\title{
Skin diseases affecting the vulva
}

Lisa Kirby MRCP is a Dermatology Specialist Registrar at Queen Elizabeth University Hospital, Glasgow, UK. Conflicts of interest: none declared.

Rosalind Simpson MRCP is an Associate Professor and Consultant Dermatologist at Centre of Evidence Based Dermatology, University of Nottingham, Nottingham, UK. Conflicts of interest: none declared.

David Nunns MD FRCOG is a Consultant Gynaecological Oncologist at Nottingham University Hospitals NHS Trust, Nottingham, UK. Conflicts of interest: none declared.

\begin{abstract}
Vulval skin disease is common in gynaecological practice. This review aims to enhance clinical skills in patient assessment, vulval examination and treatment of common benign vulval skin disease illustrated through cases. Basic treatments are often of benefit to the patient (e.g. use of emollients and topical steroids), but many patients have complex disease and can present with more than one condition so careful assessment and individualised management is essential. Understanding of when to refer onwards to a vulval specialist service is important to optimise clinical outcomes. Clinical outcomes to consider for all patients with vulval skin disease should include: (i) a reduction in symptoms (e.g. less itch, fewer flare-ups); (ii) an improvement in function (e.g. sexual function, mobility) and (iii) increased confidence in self-management (e.g. management of flare-ups and self-examination).
\end{abstract}

\section{Key words}

Vulva, lichen sclerosus, lichen planus, vulval eczema (dermatitis), psoriasis, vulvovaginal candidiasis, lichen simplex,

\section{Vulval history}

A general history should be taken but additional history points are outlines in Table 1. A detailed assessment of the presenting complaint is important, including its impact on the patient's function. Irritancy from topical treatments especially over the counter products is common. Patients commonly perceive 'low allergy' products to be safe on vulval skin but they do contain potential irritants. Enquire about current and past treatments and response. Urine is an irritant, especially to genital skin, therefore asking about incontinence is important. 


\section{Vulval examination}

A vulval examination should be carried out with the awareness of the patient's possible discomfort and embarrassment. Examination should include all areas of the vulva, perineum and perianal areas. The patient should initially be in the dorsal position for vulval and perineal examination with adequate light. Turning the patient to the left lateral position to examine the perianal area may be useful.

The normal vulva in an adult woman includes the mons pubis, inguinal folds, outer and inner labia (majora and minora), clitoris (body and hood), perineum, vestibule and anus (Figure 1). Hart's line is the junction between the vestibule and the inner labia and marks change in epithelium type from mucosal type to stratified squamous. Digital and speculum examinations of the vagina may be appropriate to look for erosions, mucosal thickening, adhesions, and scarring ${ }^{1}$. Vulval pathology may be a manifestation of a general skin condition and therefore a complete examination including the umbilicus, natal cleft, oral cavity, eyes and mouth should be performed if relevant. This allows a more complete assessment of disease extent and diagnosis especially for diseases that are not solely restricted to the vulval region such as psoriasis, eczema, lichen sclerosus and erosive lichen planus. It is important to note that the classical appearances of skin conditions in nongenital sites may not apply to the vulval area as it is a covered, moist environment. Useful terminology describing some of the lesions that may be seen in the vulval area are discussed in Table 2.

\section{Investigations}

\section{Diagnostic vulval biopsy}

A 4mm Keyes punch biopsy is adequate and should ideally be carried out under local anaesthetic at the initial visit if indicated. Rarely vulval and vaginal lesions may due to an underlying immunobullous disease. These refer to a group of blistering skin condition with an autoimmune origin. The most common of these include pemphigus vulgaris and bullous pemphigoid. If suspected then please refer to the local vulval service for further assessment. In these cases a second biopsy from perilesional skin may be needed for immunofluoroscence. It is important to avoid crushing the epithelium when taking the biopsy as this can affect histological interpretation. A vulval biopsy is usually indicated for 1), asymmetrical pigmented lesions, 2) persistently eroded areas, 3) indurated and suspicious ulcerated areas, 4) when there is poor response to treatment following the initial diagnosis.

The site selected for biopsy should be tissue-representative of the lesion or area of abnormality. 
This is usually at the edge of the lesion and should also include some normal tissue. The most central area may be inflamed or necrotic, which may give minimal tissue diagnosis because of the inflammation present.

We would recommend a diagnostic biopsy only for initial assessment. If malignancy is suspected then a $4 \mathrm{~mm}$ Keyes punch biopsy is acceptable or referral on to a gynaecological cancer team. An excisional biopsy can be problematic if the diagnosis is subsequently found to be cancer or VIN, as re-excision is often required. This can lead to further skin loss that might compromise function. Multiple mapping biopsies are indicated in cases of suspected multifocal disease to make a diagnosis and exclude invasion. This usually requires a general anaesthetic.

\section{Vaginal and vulval swabs}

Infection with candidiasis is a common cause for loss of symptom control in inflammatory dermatoses and may be a reason why lichen sclerosus appears initially well controlled with potent steroids and then flares. Minor degrees of skin trauma from scratching can produce infection. In addition, patients with diabetes may present with candidiasis. Occasionally, genital herpes can be a cause of vulval symptoms and a viral culture swab (which is different to a standard microbiological swab for bacterial culture and sensitivity) may be necessary. Genital herpes testing may not be possible in the standard gynaecology clinic so referral onto genito-urinary medicine services may be appropriate. Many patients feel antifungal treatment can help reduce symptoms but this may because of the soothing nature of the treatment that a bland emollient may give.

\section{Management}

\section{General principles}

Initial principles of management are the same for all vulval skin conditions and a holistic approach is required. Good education, support and counselling are needed with extra time given to address the disease process, discussing general vulval care measures and managing expectations. It is useful to provide information leaflets, direct patients to relevant patient-oriented websites and write down instructions for applying topical agents(see 'additional resources' for sources of patient information). The use of a mirror or model in the clinic setting is helpful to show patients where to apply their topical treatments.

\section{Correct barrier function}

Most vulval skin conditions are characterised by skin or mucosal barrier breakdown as well as underlying inflammation. Correction of the epidermal barrier is important in helping to reduce inflammation. For washing, soap and other routine cleaning agents (e.g. wipes) should be avoided, as they are likely to act as irritants or allergens. Irritation from urinary and faecal 
incontinence needs to be addressed as these are a common cause of irritation and make underlying skin pathology worse. 'Soap substitution' with a bland cream or ointment based emollient is best for cleansing. The same agent can then be used as a moisturiser to both provide a barrier to the site and sooth inflamed skin. There is no preferred emollient to use and the best one is the one the patient will adhere to. Emollient creams (not ointments) can be placed in the fridge as this can help soothe the skin.

\section{The multidisciplinary team}

Some vulval problems are chronic, rare and difficult a multidisciplinary input may be required. Service provision can vary but most hospitals in the UK provide a local vulval clinic providing expert help. Members of vulval service might include dermatology, genito-urinary medicine, physiotherapy, pain management, psychosexual therapy, pathology and urogynaecology. The individual needs of the patient will vary.

\section{Case 1}

\section{Vulval eczema}

A 40 year old female is referred with itching of the vulva. This has been getting worse for months and she finds herself scratching in her sleep, often resulting in bleeding. She had eczema as a child and gets occasional patches on the limbs when she is stressed or run down. She doesn't use any topical therapies. On further questioning she uses wet wipes to clean herself after using the toilet.

Figure 2.

Examination Poorly defined erythema of the genitocrural skin, erosions due to excoriation of the inflamed skin and white thickening (lichenification) of the labia majora due to scratching. This appearance may be due to atopic eczema, or an external cause as in contact dermatitis. There is no loss of anatomy. Examine other areas for evidence of eczema, which would typically be the antecubital and popliteal fossae.

Management: Moderate (e.g clobetasone butyrate 0.05\%) or potent (e.g. mometasone furoate $0.1 \%$ ) topical steroid plus emollients to gain control of inflammation. It is very important to ask the patient details of products used to wash and wipe the area, to help exclude contact dermatitis. The 
wet wipes may be causing local irritation in this case. It is worthwhile advising the patient to use a bland emollient as a soap substitute and to discontinue wet wipes.

Follow up: GP or dermatologist

\section{Contact dermatitis and when to think about patch testing}

Irritant contact dermatitis is common and is typically triggered by irritants eg soap, urine. Examination shows poorly defined erythema present where an irritant has been applied. Symptoms usually resolve when the irritant is removed.

Allergic contact dermatitis is a delayed type (type IV) hypersensitivity reaction to allergens in the vulval area. Common examples include topical steroids, topical anaesthetics, fragrances, sodium lauryl sulphate and topical neomycin². Clinically, the erythema may extend outside of the area where allergen has been applied. Figure 2 shows the appearance of vulval eczema that might be due to either atopic eczema or contact dermatitis.

Patch testing is a measure of delayed type (type IV) hypersensitivity reaction. It is carried out under the supervision of a dermatologist and is indicated when allergic contact dermatitis is considered. During the process, potential allergens are placed on aluminium discs and applied to the patient's back. These are removed and read at both 48 and 72 hours after application, to examine for a reaction. Allergic contact dermatitis is difficult to diagnose clinically and clinicians need to be aware of this condition when prescribing topical treatments such as steroids.

Sensitisation leading to dermatitis can occur at any time and should be considered if symptoms initially well controlled with topical therapies subsequently flare. Patients should be initially referred to a vulval service or a dermatologist for an opinion if allergic contact dermatitis is suspected. Clinical history is very important to determine potential sensitising agents which can then be applied to the skin during the patch testing process.

\section{Case 2}

\section{Vulval psoriasis}

This 60 year old lady has been referred with redness and soreness in the vulval area for many years. She uses a topical antifungal regularly which helps a little. She has a history of scalp psoriasis. 
Figure 3.

Examination: Well demarcated erythema surrounding the anogenital area with typical psoriatic plaques in surrounding skin. The genital plaque has typical scale in the perianal area, but lack of scale in the perineal and vulval areas. There is no scarring or loss of anatomy.

Clinical assessment should include examination of 'hidden sites' for other signs of psoriasis eg. knees, elbows, umbilicus, scalp, ears, lower back and nails.

Management: Moderate potency topical steroid plus emollients as recommended by NICE guidance. As the skin folds can become particularly macerated, there is a chance of secondary bacterial or fungal infection. A combination topical preparation (e.g clobetasone butyrate $0.05 \% /$ oxytetracycline $3 \% /$ nystatin) may be helpful. Biopsy if the diagnosis is unsure.

Follow up: Dermatologist or GP. Most gynaecologists may not feel comfortable in assessment and management. If so, patients should be referred on to a dermatologist.

\section{Case 3}

\section{Lichen sclerosus}

A 68 year old lady describes itching and soreness of the vulva for many years, without any discharge. Her symptoms flare up intermittently and sometimes she finds underwear very uncomfortable. She has been treated for thrush several times by the GP, but her symptoms never fully clear. She finds the issue very embarrassing. She admits to washing the area three times a day with shower gel because she feels unclean.

Figure 4.

Examination: This is advanced lichen sclerosus (LS) with loss of normal anatomy (scarring). There are porcelain white papules and plaques and occasional ecchymoses (purpura). A typical early finding is 'figure of 8' appearance of erythema involving the vulval and perianal region. However, LS is often diagnosed quite late, therefore late findings of loss of normal anatomy, labial fusion and adhesions may be present at the first consultation. Occasionally papery white plaques can be found on other areas of the body. It is very unusual for LS to affect the mouth. The clinical findings are often adequate to establish a diagnosis. However, biopsy may need to be considered if there are any indurated areas or areas suspicious for VIN or malignancy.

\section{Management:}


A recent Cochrane systematic review showed there is reasonable randomised controlled trial evidence for the use of very potent topical steroids in lichen sclerosus ${ }^{3}$. The British Association of Dermatologists suggest the use of the very potent topical steroid, clobetasol proprionate $0.05 \%$ (Dermovate), over a three-month reducing course (suggested daily for one month, alternate days for month and twice a week maintenance $)^{4}$. Long term maintenance with twice weekly therapy is recommended and the patient will use 30-60 g topical steroid per year. Restoration of barrier function with a bland emollient is essential. This can be used as often as needed and can be used as a soap substitute. Posterior fourchette fissuring causing dyspareunia may require digital massage of the steroid into the fissure. Vaginal dilators and lubricants may also be useful. There is a small risk of cancer (less than $5 \%$ risk) so patients should be encouraged to self-examine on a regular (suggested monthly) basis. This patient should also be told to wash only once a day. Overwashing may perpetuate symptoms.

Follow up: Gynaecologist, dermatologist or GP. Refer to vulval service for treatment resistant cases, complications of treatment, difficult disease and when associated with VIN.

\section{Use of topical steroids on the vulva}

Topical steroids reduce inflammation associated with skin diseases such as lichen planus, lichen sclerosus and eczema leading to improvement in symptoms and appearance. However, there are patient and physician concerns at the use of topical steroids due to worry about side-effects, particularly skin or mucosal atrophy, which can lead to under-treatment and less control of symptoms. It is important therefore to use the correct strength of topical steroid for the necessary length of time on the appropriate body site with the clear instructions to patient so that they can be confident in self-management and be reassured that when applied correctly 'skin thinning' is very unlikely and may occur when applied to the wrong area. Mucosal surfaces such as the vulval vestibule eg in lichen planus (see below) are remarkably resistant to steroid atrophy.

In general, topical steroids should be used once-daily. There is no evidence to suggest that twicedaily application is superior, although twice-daily has greater potential to cause side effects. Ointments are preferable to creams as they contain fewer constituents and therefore have a lower chance of causing irritation/contact allergy. Once control of inflammation and symptoms has been achieved, topical steroids should be reduced to the minimum frequency required to maintain remission. The concept of 'weekend therapy', that is, applying topical steroids on two consecutive days per week, is effective in atopic eczema patients and can extrapolated to chronic vulval diseases such as lichen sclerosus and lichen planus where long-term maintenance therapy is required. Topical steroids should only be used on affected areas to prevent side-effects in 
adjacent skin. Box 1 outlines reasons why there may be a failure to respond to topical steroid treatments.

\section{Case 4}

\section{Erosive lichen planus with oral involvement}

A 30 year old female is referred with a longstanding history of vulval soreness and dyspareunia. She mentions that smears are very difficult. She uses emollients which help but her condition never settles. She also describes a sore mouth, for which she sees the dentist regularly. Figure 5, figure 6.

Examination findings Two types of lichen can affect the vulva; 'classical' lichen planus gives violaceous, well demarcated plaques with overlying lacy white lines which usually affect the labia majora and surrounding skin. This can also affect extra-genital sites. Erosive lichen planus (as seen here) may present with 'glazed' erythema or erosions symmetrically distributed at vaginal introitus. Lacy white lines (Wickham's striae) may be seen in surrounding skin and also in the buccal mucosa. There may be loss of anatomy. These patients can have vaginal involvement with significant vaginal stenosis so speculum examination is important.

Management: There are currently no specific guidelines for lichen planus, but the standard practice is to use a similar regimen as for lichen sclerosus and case series evidence supports this ${ }^{5}$.The response rate to treatment is lower than for lichen sclerosus and there is a place for systemic therapies such as methotrexate in those with severe, recalcitrant or multisystem disease.

Follow up: Dermatologist or gynaecologist. For classical type refer to vulval service for treatmentresistant cases and when associated with VIN. Erosive lichen planus is difficult to treat so refer early to a vulval service. Consider referral to oral medicine if significant oral involvement. 


\section{Conclusion}

Core competencies in vulval disease for the general gynaecologist should include being able to take a detailed patient history and examination, make a diagnosis and starting basic treatment. It is important to underpin the treatments with clear explanation to the patient on vulval care and the use of topical steroids to improve clinical outcomes. Patients with complex or rare conditions, or those who are treatment resistant, should be referred to a vulval service.

\section{Practice points}

- Take a thorough symptom and function history and perform a systematic examination

- Describe vulval conditions in dermatological terminology to support the diagnosis

- Avoid empirical prescriptions before there is clinical evidence to support the diagnosis. If you are not sure of the diagnosis please ask for advice

- Provide written information and clear instructions to the patient where possible especially with regards the use of topical corticosteroids

- Employ a low threshold for a vulval biopsy if there is any doubt about the diagnosis

\section{References}

1.Schlosser BJ, Mirowski GW. Approach to the patient with vulvovaginal complaints. Dermatol Ther. 2010;23(5):438-48. doi: 10.1111/j.529-8019.2010.01348.x.

2. O'Gorman SM, Torgerson RR Allergic contact dermatitis of the vulva. Dermatitis. 2013 MarApr;24(2):64-72.

3. Chi CC, Kirtschig G, Baldo M, Brackenbury F, Lewis F, Wojnarowska F. Topical interventions for genital lichen sclerosus. Cochrane Database Syst Rev. 2011(12):CD008240. doi:10.1002/14651858.CD008240.pub2. 
4.Lewis FM, Tatnall FM, Velangi SS et al. British Association of Dermatologists guidelines for the management of lichen sclerosus, 2018.Br J Dermatol 2018;178(4):839-853. doi:

10.1111/bjd.16241

5.Simpson RC, Littlewood SM, Cooper SM, Cruickshank ME, Green CM, Derrick E et al. Real-life experience of managing vulval erosive lichen planus: a case-based review and U.K. multicentre case note audit. The British journal of dermatology. 2012;167(1):85-91. doi:10.1111/j.13652133.2012.10919.x

\section{Further resources;}

Dermnetnz: The Dermatology Resource www.dermnetnz.org

British Society for the Study of Vulval Disease www.bssvd.org

Online resource endorsed by the ISSVD http://vulvovaginaldisorders.com/

Online training course which is free to UK doctors to access

www.e-lfh.org.uk/projects/dermatology/index.html

\section{FIGURES}

Figure 1: Schematic representation of the normal adult vulva. Copyright "Dawn Danby and Paul Waggoner", c/o ISSVD 
Figure 2: Vulval eczema (dermatitis). Note poorly defined erythema of the genitocrural skin, erosions due to excoriation in the inflamed skin and white thickening (lichenification) of the labia majora due to scratching. This appearance may be due to atopic eczema, or an external cause as in contact dermatitis (either irritant or allergic contact dermatitis).

Figure 3: Psoriasis affecting the female genitalia. Well demarcated erythema surrounding the anogenital area with typical psoriatic plaques in surrounding skin. The genital plaque has typical scale in the perianal area, but lack of scale in the perineal and vulval areas.

Figure 4: Advanced vulval lichen sclerosus showing ecchymoses, atrophy and loss of normal anatomy.

Figure 5: Desquamative gingivitis with evidence of Wickham's striae in the buccal mucosae. Courtesy of Dr Ruth Murphy.

Figure 6: Vulval erosive lichen planus 

Table 1 - vulval history-taking points

\begin{tabular}{|c|c|}
\hline Question & Reasoning \\
\hline $\begin{array}{l}\text { What are the key symptoms and how } \\
\text { severe are they? }\end{array}$ & $\begin{array}{l}\text { Important to be clear on the initial symptom. Itch can suggest skin disease or infection. Pain } \\
\text { can be secondary to itching from skin damage through trauma. Vaginal discharge may suggest } \\
\text { infection. }\end{array}$ \\
\hline $\begin{array}{l}\text { How long has the woman been } \\
\text { experiencing symptoms? }\end{array}$ & $\begin{array}{l}\text { Acute symptoms may indicate infections such vulvovaginal thrush or contact dermatitis. } \\
\text { Chronic symptoms may be due to lichen sclerosus or lichen planus. }\end{array}$ \\
\hline $\begin{array}{l}\text { What is the impact on the patient's } \\
\text { function? ('How do the symptoms affect } \\
\text { you?' or 'What do you miss as a result of } \\
\text { the problem?') }\end{array}$ & $\begin{array}{l}\text { Improvement in function (including sex) is an important clinical outcome. Functional impairment } \\
\text { should be documented at every consultation }\end{array}$ \\
\hline $\begin{array}{l}\text { What treatments have been tried before } \\
\text { (including over the counter agents)? }\end{array}$ & $\begin{array}{l}\text { The history should explore failed treatments e.g. topical steroid frequency and amount as under } \\
\text { usage with these treatments is common due to steroid phobia or a lack of understanding by the } \\
\text { patient. Inappropriate topical treatments can exacerbate symptoms and potentially cause an } \\
\text { irritant reaction. }\end{array}$ \\
\hline $\begin{array}{l}\text { How is the patient cleaning the vulval } \\
\text { area? }\end{array}$ & Over-washing may lead to skin damage and further irritation. \\
\hline $\begin{array}{l}\text { Are there any possible contacts with } \\
\text { irritants e.g. soaps, shampoos, urine, and } \\
\text { scented vaginal wipes? }\end{array}$ & $\begin{array}{l}\text { These are potential irritants and can damage the skin potentially causing inflammation. Urine } \\
\text { and scented vaginal wipes are potent skin irritants. }\end{array}$ \\
\hline Are symptoms stress related? & $\begin{array}{l}\text { In lichen simplex, itching is classically worse at times of stress. Many inflammatory skin } \\
\text { conditions may flare during stressful life events. }\end{array}$ \\
\hline Is there any systemic illness? & $\begin{array}{l}\text { For example, diabetes, renal failure, anaemia (these may all be a cause of itch), or } \\
\text { autoimmune conditions (higher chance of systemic autoimmunity in lichen sclerosus or erosive } \\
\text { lichen planus). A family history of autoimmunity should also be asked..= }\end{array}$ \\
\hline $\begin{array}{l}\text { Are there any other skin conditions } \\
\text { present? }\end{array}$ & $\begin{array}{l}\text { Skin disease at other skin sites may provide clues to the vulval diagnosis. For example, } \\
\text { eczema or psoriasis. These may be very obvious, however signs may be subtle, for example } \\
\text { psoriasis is sometimes hidden as cracking behind the ears, a scaly scalp or umbilical } \\
\text { erythema) }\end{array}$ \\
\hline
\end{tabular}

Table 2: Terminology of lesions that may be seen in the vulval area 


\begin{tabular}{|c|c|c|}
\hline $\begin{array}{l}\text { Lesion } \\
\text { terminology }\end{array}$ & Description & Example \\
\hline Fissure & $\begin{array}{l}\text { A thin 'hairline' crack in the skin surface due to excessive } \\
\text { dryness }\end{array}$ & Psoriasis and lichen sclerosus \\
\hline Excoriation & Scratch mark, may be single or multiple & $\begin{array}{l}\text { May be seen in any itchy skin } \\
\text { condition e.g. atopic eczema, } \\
\text { lichen sclerosus }\end{array}$ \\
\hline Erosion & $\begin{array}{l}\text { A shallow denuded area due to loss of the epidermis (surface } \\
\text { layer of skin) }\end{array}$ & Erosive lichen planus \\
\hline Ulcer & Full thickness loss of the epidermis (top layer of skin) $+/$ - dermis & Aphthous ulceration \\
\hline Macule & Flat area of colour change & $\begin{array}{l}\text { Vulval melanosis } \\
\text { Ecchymosis (subcutaneous } \\
\text { purpura) seen in lichen } \\
\text { sclerosus }\end{array}$ \\
\hline Nodule & Large palpable lesion greater than $0.5 \mathrm{~cm}$ in diameter & $\begin{array}{l}\text { Squamous cell carcinoma, } \\
\text { Scabies }\end{array}$ \\
\hline Papule & Small palpable lesion less than $0.5 \mathrm{~cm}$ in diameter & $\begin{array}{l}\text { Genital warts } \\
\text { Molluscum contagiosum } \\
\text { Seborrhoeic keratosis }\end{array}$ \\
\hline Plaque & $\begin{array}{l}\text { A palpable flat lesion greater than } 0.5 \mathrm{~cm} \text { diameter. It may be } \\
\text { elevated or may be a thickened area without being visibly raised } \\
\text { above the skin surface }\end{array}$ & $\begin{array}{l}\text { Vulval Intraepithelial Neoplasia } \\
\text { Squamous cell carcinoma } \\
\text { Large seborrheic keratosis }\end{array}$ \\
\hline Vesicle & Small fluid filled blister less than $0.5 \mathrm{~cm}$ diameter & Bullous pemphigoid \\
\hline Lichenification & $\begin{array}{l}\text { An accentuation of skin markings commonly associated with } \\
\text { thickening of epidermis usually caused by scratching or rubbing }\end{array}$ & Lichen simplex \\
\hline
\end{tabular}


Box 1 - Reasons why there may be a failure to respond to topical steroid treatments within vulval skin conditions

- Continued exposure to irritants - eg urine or faeces, external products such as wipes or non-prescribed topical treatments and over washing with water can all contribute towards irritation and ongoing symptoms.

- Poor adherence to prescribed treatment regimen -The patient should be advised to apply the topical steroid in terms of the fingertip unit (A fingertip is from the very end of the finger to the first crease in the finger. It does NOT mean a blob on the fingertip). The number of fingertip units required is usually one to two but is specifically tailored to the patient depending upon surface area affected by the condition.

- Inaccurate placement of topical steroid - the patient may be applying the topical treatment to an unaffected area. Especially common if the patient is elderly and unable to use a mirror to see what they are doing.

- Incorrect diagnosis - if adherence and skin care practices are assessed as adequate, it may be that the diagnosis given is incorrect. An allergic contact dermatitis to topical treatments may have occurred (see section on Patch Testing) or there may be pre-malignant or malignant change in the affected area. If there is any concern a biopsy should be taken

- An inappropriate and/or weak steroid has been prescribed eg $1 \%$ hydrocortisone. Reducing the potency of the steroid ointment is not usually practiced and it is more usual to reduce the frequency of application of the very potent steroid.

- Superimposed infection - eg candidiasis. 
